\title{
Conférence d'Hydraulique de la Société Hongroise d'Hydrologie et de I'Institut Hongrois de Recherches des Ressources Hydrauliques
}

Budapest, 5-10 septembre 1960

\author{
The Hydraulics Conference \\ of the Hungarian Hydrological Society \\ and the Hungarian Institute for Research \\ on Hydraulic Resources held at Budapest \\ from the 5th-10th September 1960
}

PAR

Le Professeur J. NOUGARO

I.AURÉAT DE L'INSTITUT,

DIRECTEUR TECHNIOUE

DE L'INSTITUT DE MÉCANIQUe DES FLUIDES DE TOULOUSF

ET

In Professeur GERBER

lahuréat de l'isstitut,

Principanx sujets traités au cours de la Conférence :

- Hydraulique fluviale (débit solide et mouvement de l'ean);

- Hydraulique de la filtration (infiltration dans les conditions naturelles et infiltration en dessous d'ouvrages).
The following were the main subjects discussed at the Conference:

1) River hydraulics (sediment load and water movement)

2) The hydraulics of filtration (infiltration under natural conditions and underneath man-made structures).
Une «Conférence d'Hydraulique », organisée par la Société Hongroise d'Hydrologie et l'Institut de Recherches des Ressources Hydrauliques, s'est tenue à Budapest du 5 au 10 septembre 1960 sous la présidence du professeur $\mathrm{D}^{\mathrm{r}} \mathrm{E}$. Nemeth, président de la Commission de l'Economie Hydraulique de l'Académie des Sciences de Hongrie.
Les deux sujets traités, au cours de la Conférence, sont les suivants :

- Hydraulique fluviale;

- Hydraulique de la filtration.

Les études relatives à l'Hydraulique fluviale sont réparties en deux groupes : 
Groupe I : Débit solide;

Groupe II : Mouvements d'eau.

Les études correspondant à l'Hydraulique de la filtration ont également été réparties en deux groupes :

Groupe III : Infiltration dans les conditions naturelles;
Groupe IV : Infiltration au-dessous d'ouvrages d'art et à travers les digues. Hydraulique des puits.

L'objet de la présente communication est de rapporter un bref résumé de l'ensemble des rapports qui ont été présentés au cours de ces journées d'études.

\section{I. - HYDRAULIQUE FLUVIALE}

Les éludes correspondantes, au nombre de 36, ont été réparties dans les deux groupes suivants :

- Groupe I : Débit solide (18 rapports dont 6 hongrois).

Rapporteur général: M. BoGaRDI, docteur ès Sciences Techniques, chef de Section de l'Institut de Recherches des Ressources Hydrauliques.

- Groupe II : Mouvements d'eaux (18 rapports dont 5 hongrois).

Rapporteur général: M. Laszloffy, docteur ès Sciences techniques, chef de la Section Eaux de Surface de l'Institut de Recherches des Ressources hydrauliques.

Dans cette communication, nous ferons un bref résumé des principales études présentées, la liste complète des publications étant jointe à l'annexe de la présente note.

\section{Groupe I : Débit solide.}

\section{1 . Etudes relatives aUX problèmes géNÉraUX DU DÉBIT SOLIDE.}

Le premier des rapports présentés traite de l'étude de la régularisation d'une rivière en se basant sur une analyse morphologique. Le but de la communication est de préciser les moyens pour stabiliser un lit à la suite de rupture des rives. L'auteur détermine différentes relations, principalement entre les profondeurs d'eau et les rayons de courbure, et montre quels sont les travaux à assurer pour un équilibre relatif des éléments hydrauliques.

La deuxième étude se rapporte aux lois générales de la formation du débit solide des rivières. Cette étude est basée sur la détermination de ces transports et tenant compte de toutes les particularités du bassin versant. Une équation per- met de définir, pour chaque profil, la quantité de débit solide qu'il traverse. Une application à une rivière roumaine montre que l'erreur obtenue est admissible pour les besoins de la pratique.

La troisième étude essaie de définir les rapports entre les caractéristiques physiques de cours d'eau transportant des matières solides. Par exemple : pour des cours d'eau à lit mobile l'auteur s'efforce de définir des formules de vitesse dans lesquelles interviennent différents paramètres : état du lit, quantité de débit solide, etc... L'étude fournit plusieurs exemples et ouvre la voie à des recherches futures dans ce domaine.

Le quatrieme rapport précise quelle est l'influence de l'envahissement d'un lit sur les herbes et l'envasement des cours d'eau. En tenant compte de ces paramètres, l'auteur détermine la relation entre la vitesse et la hauteur d'eau et constate que, par suite de la végétation en particulier, la vitesse moyenne subit des changements importants. De ce fait, le cours d'eau devient incapable d'assurer le transport des grains de débit solide dépassant une certaine dimension.

\section{$2^{\circ}$ Etudes Relatives aU TRANSPORT SOLIDE EN SUSPENSION.}

Le premier rapport se rapporte à des problèmes généraux sur la théorie de la suspension. L'étude fournit en particulier une équation relative à la répartition de la concentration suivant la profondeur, en insistant sur la difficulté de définir cette concentration dans les couches situées au fond de la rivière.

La deuxième étude est relative également aux relations pouvant exister entre la vitesse des courants et la hauteur d'eau. En partant de lois physiques connues et tout particulièrement des relations de Prandtl, l'auteur déduit, sur la base de la vitesse de glissement, une relation entre la longueur sur laquelle s'opère le mélange de 
débit solide et la rugosité. Il conclut que la vitesse de glissement exprime, en définitive, une loi de résistance. L'étude est également développée dans le cas d'une rivière à couverture de glace.

La troisieme communication traite du mouvement du débit solide des suspensions dans un écoulement d'eau variable. Après un rappel de la theorie du transport turbulent de débit solide, les auteurs déterminent des équations de transport du débit solide basées sur la méthode des différences finies. Ils aboutissent ainsi à une simplification de calcul, favorisée par une linéarisation des équations. La méthode peut aussi être utiliséc soit par relaxation, soit avec machine à calculer digitale.

Le quatrième rapport fournit un résultat de mesure de turbulences dans un canal vitré à l'aide d'une méthode cinématographique. L'auteur précise que cette méthode, malgré l'inconvénient des prises de vues qui constituent un travail particulierement long, convient fort bien pour l'étude du phénomène précité.

La cinquième étude est une contribution à la théorie du transport solide basée sur le principe de la diffusion. Après une critique de cette théorie, l'auteur présente des équations différentielles valables aussi bien en mouvement permanent qu'en régime non permanent.

Les conclusions expriment l'opinion que cette théorie est parfaitement valable dans le cas d'un débit solide argileux ou limoneux.

\section{$3^{\circ}$ ETUDEs TRAITANT DES ESSAIS SUR MODÉLE RÉDUTT.}

La première communication est relative à la présentation de trois essais en laboratoire au sujet de l'aménageement d'un cours d'eau. Au cours de ces expériences, l'auteur analyse l'influence du nombre de Reynolds, du diamètre des grains, de la concentration, etc.

La deuxième étude se rapporte à un modèle établi pour étudier le débit solide en suspension dans une retenue primaire. Les essais relatés permettent, en particulier, de préciser les éléments suivants :

- Détermination du débit solide;

- Détermination de la répartition des vitesses dans le bassin;

-. Dispositif en vue d'assurer une sédimentation uniforme.

L'auteur propose, en outre, une formule de calcul du débit solide et note que sa théorie fournit des résultats plus satisfaisants que les formules classiques de Schoklitsch, Casey, MeyerPeter, et Shields.

La troisième communication est un rẻsumé général des études sur modèle réduit entreprises pour des affouillements de lits de rivières. Les expériences réalisées permettent à l'auteur de définir le paramètre qui joue un rôle principal dans l'affouillement des lits : la vitesse moyenne, la densité de l'eau, le débit solide, la víscosité cinématique, la grosseur des grains, la répartition granulométrique, le transport $d u$ débit solide, roulé, etc.

L.e quatrième rapport relate des expériences relatives à la forme des lignes de courant dans des cours d'eau naturels. Bien que ces études n'aient pas été terminées au moment du Congrès, l'auteur avance quelques conclusions basées sur ses observations personnelles. Il note, en particulier, l'influence des courbes sur le rétrécissement et le changement des profils. Il précise linfluence du nombre de Froude. Ces investigations paraissent fort intéressantes car elles doivent conduire à la détermination d'une distorsion admissible dans les études sur modèle réduit de travaux de régularisation de rivières.

\section{$4^{\circ}$ Etunes Relatives aUX PROBLEMES DE L'ENVA- SEMENT DES BASSINS ET AUX PHÉNOMÉNES DF. InA SÉDIMENTATION.}

Le premier rapport traite de la distribution du débit solide en suspension dans un écoulement variable. Après un rappel des méthodes thériques existantes, l'auteur recommande d'utiliser une méthode de différences finies. Il exprime ainsi la répartition de la concentration après certaines transformations avec une fonction ressemblant à celle de Green. Cette étude semble constituer un essai très intéressant pour déterminer le transport de débit solide dans un monvement d'eau variable.

La deaxième communication se rapporte à une méthode de calculs des dépôts dans les bassins de décantation. L’auteur expose dans cette étude une méthode rapide à l'aide de laquelle on peut déterminer, pour un cours d'eau donné, la proportion de débit solide transporté en suspension. et du débit solide se décantant dans un bassin de retenue à profil variable.

Le troisième rapport traite de l'envasement d'une retenue en Hongrie. Ces recherches ont trait aux modifications du lit et à l'envasement se produisant à l'amont d'une chute aménagée effectivement dans une rivière.

Les auteurs arrivent ì la conclusion que l'enva- 
sement le plus dangereux se produit, en général, dans un secteur situé à la limite du remous provoquée par l'installation.

\section{$5^{\circ}$ Etudes RElatives AUX Problèmes de L'Éva- CUATION HYDRAULIQUE DES DÉPÔTS.}

Le premier rapport traite d'une méthode simplifiée pour le calcul de l'évacuation des dépôts charriés dans une retenue. L'auteur expose en détail quelles sont les données qui doivent être réunies pour un tel calcul : paramètre relatif au lit stabilisé de la retenue, choix du débit de refoulement, paramètre relatif au nettoyage hydraulique de la retenue. Pour le calcul de l'évacuation hydraulique proprement dite, l'auteur recommande une méthode basée sur le fait que la pente du fonds ne varie pas pendant l'opération. En modifiant légèrement cette méthode, il est possible de tenir compte précisément de cette variation de fonds.

La deuxième étude se rapporte à l'évacuation hydraulique des dépôts charriés dans une retenue. Tandis que la première étude se rapportait à l'évacuation de la matière charriée déjà déposée, ce deuxième rapport traite de l'évacuation hydraulique des ouvrages de prélèvement d'eau et de dessableurs. L'étude est principalement con. sacrée à l'évacuation périodique de ces dépôts avec des pertes d'eau minimes. Cette étude paraît particulièrement importante. Les auteurs appuient leurs constatations sur des exemples pratiques.

\section{GROUPE II : Mouvements d'eau.}

\section{1" QUESTION FONDAMENTALE D'HYDRAUliQue. FLUVIALE.}

Deux études traitent de la résistance apportée aux mouvements de l'eau par le lit d'une rivière.

La première fournit des résultats d'expériences qui conduisent leurs auteurs à préciser que les formules de vitesse modernes supposant une loi de frottements logarithmiques sont exactes.

La deuxième traite du rayon hydraulique et de son ròle dans le calcul du débit. L'auteur critique tout d'abord l'appellation de rayon hydraulique et trouve qu'il serait plus correct de l'appeler « profondeur moyenne ». II insiste sur les difficultés de la détermination du rayon hydraulique dans le cas de profils complexes de cours d'eau. Les problèmes généraux du mouvement de l'eau sont encore traités dans unc troisième étude qui a trait au rapport entre les divers régimes d'écoulement et la vitesse des ondes. Cette étude est relative à une comparaison entre la valeur de la vitesse de propagation d'une onde et la valeur de la vitesse moyenne du courant. Elle est complétée par des constatations sur la vitesse critique d'un écoulement.

Une quatrième étude est relative à l'introduction dans les calculs hydrauliques de profils en long qui représentent les données hydrauliques des cours d'eau naturels. Parmi ceux-ci, par exemple, les lignes de niveau relatif à un débit constant constituent un élément très important pour le contrôle des changements de lits intervenant sous l'effet de travaux de régularisation. La représentation dans un profil des surfaces de la largeur de la pente superficielle, de la profondeur, de la vitesse moyenne, de la débitance, facilite le calcul de toutes les lignes d'eau.

\section{$2^{\circ}$ Etude RELATIVE A L'ÉCOULEMEN'T PERMANENT GRADUELLEMENT VARIÉ.}

Un seul rapport est présenté dans cette partie. Il a trait à l'intégration de l'équation différentielle de l'écoulement permanent graduellement varié pour lits réguliers prismatiques ou cylindriques. Il est certain que les méthodes graphiques ou numériques sont plus avantageuses que les procédés analytiques.

\section{$3^{\circ}$ Problème relatif a L'Écoulement Non per- MANENT.}

C'est certainement la partie la plus importante de ce groupe qui a trait au domaine de l'écoulement non permanent.

La première étude se rapporte à l'élaboration d'une nouvelle méthode grapho-analytique pour le calcul de ces mouvements. C'est la transformation d'une méthode de calcul des courbes de remous qui a été transformée. Elle procède par approximations successives. Des expériences actuelles sont en cours pour valider la méthode proposée.

Le deuxième rapport est relatif à la méthode de calcul numérique que nous avons eu nousmêmes l'occasion de présenter et qui a fait l'objet, dans ses développements. d'une communication devant le Comité Technique de la S.H.F.

La troisième étude se rapporte à l'utilisation de valeurs adimensionnelles pour la propagation des ondes. L'introduction du nombre de Froude comme paramètre de base permet d'obtenir des résultats particulièrement intéressants pour les 
ondes de petite amplitude. Pour des ondes d'amplitude plus importante, les résultats ne sont applicables qu'en admettant une différence de l'ordre de $25 \%$. L'auteur aboutit à des abaques qui ne sont pas actuellement achevés.

Le quatrième rapport est relatif à l'étude des ondes de choc par application directe des formules de calcul des ondes de choc dans les conduites à canaux découverts.

La cinquième étude rend compte des procédés par lesquels il est possible de déterminer la transformation d'une onde de crue traversant un bassin de retenue. L'auteur fournit de nombreuses solutions parmi lesquelles une paraît particulièrement simple pour résoudre, avec une certaine approximation, la question.

La sixième communication est relative à la détermination du débit nécessaire pour calculer les évacuateurs de crue des lacs d'accumulalion. Sur la solution de l'équation de continuité relative à un bassin plein qui a été utilisée, les auteurs cherchent une solution analytique de cette équation, d'une part par linéarisation, d'autre part par un procédé d'approximation successive. Cette étude doit être complétée par des documents qui n'ont pu être joints à la communication.

La septième étude est relative à une propagation d'ondes de crue artificielles sur un petit ruisseau. Son but est d'éclaircir le rapport entre la vitesse de propagation et la vitesse moyenne. S'inspirant d'études connues, son auteur applique les résultats à trois essais qui ne peuvent, il le précise lui-même, être généralisés mais qui conduisent à une voie pouvant être utilisée pour des essais analogues.

\section{$4^{\circ}$ Etudes diverses d'hydraulique.}

La première étude présentée par notre laboratoire est relative à l'étude d'un prototype semiindustriel de barrage déversoir à fente aspiratrice. Cette étude a fait également l'objet d'une communication à la S.H.F. et a été ensuite publiée dans la revue La Houille Blanche.
Le deuxième rapport a trait à l'influence des distorsions géométriques et hydrauliques dans des modèles de dérivations à surface libre. Les résultats des essais montrent le rôle prépondérant du nombre de Reynolds et précisent les limites admissibles de la distorsion.

Le troisième rapport est consacré à l'entraînement d'air dans les courants d'eau très rapides. L'atuteur expose, en particulier, les procédés servant à assurer la teneur en air du mélange eauair, ainsi que les vitesses et débits.

Il étudie l'influence de l'entraînement d'air dans la couche limite, illustrant l'exposé de nombreux résultats d'expériences.

\section{5 "Etudes D'hydrologie.}

La première de trois études s'occupe du renforcement des débits maximaux dans les lits fluviaux. Cette étude extrêmement importante laisse de côté le cas particulièrement connus, pour s'attacher aux crues extraordinaires dites «murs d'eau » ou encore « ondes de crue » montant avec une très grande rapidité à une hauteur particulièrement importante. Ces crues sont observées lors de pluies torrentielles dans des lits temporairement secs.

la denxième étude donne une méthode de détermination du volume d'eau des ondes de crue des petits bassins versants. L'auteur propose en particulier une formule permettant de déterminer le débit de crue en fonction du débit maximal. Il propose également des abaques simples pour le calcul de la durée de passage du débit de l'onde de crue dépassant une certaine valeur.

Enfin, la troisième étude présente un emploi des nomogrammes dans les prévisions hydrologiques. L'auteur prouve, à l'aide d'une série d'abaques, définis par lui, combien la nomographie peut fournir une aide précieuse dans le travail de routine et de prévision des crues : une règle à calculer construite sur cette base fournit très rapidement des résultats particulièrement intéressants. 


\section{II. - HYDRAULIQUE DE LA FILTRATION}

Les études correspondantes, au nombre de 31 dont 19 hongroises, ont été réparties dans les deux groupes suivants :

- Groupe III : Infiltration dans les conditions naturelles (12 rapports, dont 10 hongrois).

Rapporteur général : M. IHRIG, chef de la Section Hydraulique Souterraine à l'Institut de Recherches des Ressources hydrauliques.

-- Groupe IV : Infiltration au-dessous d'ouvrages d'art et à travers les digues, hydraulique des puits (19 rapports dont 9 hongrois).

Rapporteur général : M. Mosonyı, membre correspondant de l'Académie des Sciences de Hongrie.

Nous allons passer rapidement en revue les principaux résultats exposés dans les études présentées.

\section{GROUPE III : Infiltration dans les conditions naturelles}

\section{$1^{\circ}$ Filtration en sol Saturé (2 rapports).}

Le premier des deux rapports présentés traite de l'écoulement plan à surface libre se produisant à travers les digues en terre.

Le second travail expose les résultats expérimentaux obtenus sur les limites supérieure et inférieure de validité de la loi de Darcy :

-- La limite supérieure est étudiée comme il est d'usage en fonction du nombre de Reynolds, la dimension caractéristique choisie étant le diamètre efficace des grains. Vérifiée pour $\mathcal{R}<5$, la loi linéaire s'écarte ensuite de plus en plus de la réalité lorsque $\mathcal{R}$ augmente. On constate alors, parallèlement aux effets de l'inertie, l'apparition d'une turbulence qui paraît rester locale jusqu'à $\mathcal{O}<100$ pour s'étendre ensuite à tout le domaine d'écoulement. La loi de perte de charge prend alors la forme :

$$
\mathrm{V}=\mathrm{CJ}^{0,74}
$$

- La limite inférieure est définie en fonction d'un gradient de charge critique $\mathrm{J}_{c}$ au-dessus duquel la loi linéaire est vérifiée. Si $\mathbf{J}<\mathrm{J}_{c}$, la vitesse réelle est inférieure à la vitesse de Darcy. Ce phénomène subsiste dans les sols argileux, mais le début du mouvement se pro. duit pour une valeur $\mathrm{J}_{0}$ du gradient $0<\mathrm{J}_{0}<\mathrm{J}_{\text {c }}$. d'autant plus élevée que la teneur initiale en cau est faible. Pour les échantillons étudiés, $\mathrm{J}_{0}=0$ pour une teneur en eau de $42,5 \%$.

2" MOUVEMENT EN SOL NON SATURE (3 rapports).

La première étude, hydropédologique, rend compte d'essais sur échantillons de sol naturel, ct tente une interprétation des variations de perméabilité observées en fonction des cations absorbés, des propriétés chimiques et mécaniques des sols.

Le second problème traité est celui des « phénomènes transitoires provoqués par des ondes de crue dans les couches aquifères au voisinage des lits de rivières ». Certaines ruptures du sol situces à l'aval des digues de protection ne sont pas dues à l'entraînement du terrain par « renard », mais à la compressibilité notable du mélange eau-air se mettant en mouvement au début de la propagation de l'onde de crue et à l'accroissement de pression des pores en résultant.

La frange capillaire existant dans l'écoulement à surface libre vers un puits est enfin étudiée dans un travail expérimental important mettant en lumière les deux points suivants :

- Débit circulant dans la frange capillaire. Ce débit est mesuré, une formule empirique est proposée pour son calcul;

- Influence de la remontée capillaire sur la réalisation des modèles réduits, les phénomènes capillaires n'obéissant pas aux mêmes lois de similitude que l'écoulement saturé.

\section{3" Eatx artésiennes (1 rapport).}

La théorie des eaux artésiennes ne permettant pas de rendre compte dans de nombreux cas de la montée des eaux observée dans les forages, le seul rapport présenté sur ce sujet « Théorie de la Géohydrostatique », propose d'expliquer ce phénomène par une tension supplémentaire à laquelle serait soumise l'eau des couches profondes. Cette tension supplémentaire est attribuce à la compression élastique des sédiments perméabīes.

Il existerait alors trois sortes de pressions :

- La pression géologique;

-- la pression hydrostatique;

- Ia tension supplémentaire, due à la compres. sion supplémentaire géohydrostatique, assurant l'élévation de l'eau dans les forages. 
Il en résulterait que, dans de nombreux cas, l'exploitation de telles nappes constitue une consommation de réserves sans possibilité de reconstitution.

\section{4" Application a L'irrigation (4 rapports).}

Dans ce paragraphe, sont classés les sujets suivants :

- Etude de la répartition du débit infiltré audessous des rizières en fonction de la perméabilité du sol, des dimensions des planches et de la profondeur de la couche imperméable. On constate en général une infiltration plus élevée sur les limites qu'au centre.

- Pertes par infiltration dans les canaux d'irrigation : comparaison entre les diverses formules existantes et des mesures in situ. Proposition d'une nouvelle relation :

$$
\mathrm{Q}_{t}=\mathrm{Q}_{0} \mathrm{HP}_{r}
$$

$Q_{t}$ Débit en mètre courant du canal;

$H$ Tirant d'eau;

$P_{r}$. Périmètre mouillé;

$Q_{0}$ Débit d'infiltration d'un tube de mesure normalisé battu à une profondeur de $30 \mathrm{~cm}$.

- Etude expérimentale de l'irrigation par ruissellement permettant de définir les caractéristiques rationnelles de ce type d'irrigation. Une théorie qui rend compte dans de bonnes conditions des expériences réalisées est ensuite proposée.

- Une dernière communication, qui n'est pas une étude hydraulique, examine les facteurs qui influencent les pertes des canaux d'irrigation, dans les conditions existant en Hongrie.

\section{$5^{n}$ INFLUENCE DES FACTEURS NATURELS (2 rapports).}

Un premier rapport de synthèse présente les eaux souterraines de la Roumanie et caractérise l'abondance en eau par un indice de sécheresse.

$$
k_{a}=\frac{\text { évaporation }}{\text { précipitation }}
$$

Les études hydrogéologiques ont toujours à considérer. des couches de perméabilité el d'épaisseur variables. Pour définir d'une manière plus précise tout problème hydrogéologique, le second rapport présenté propose d'introduire, en complément de la perméabilité moyenne, deux coefficients d'irrégularité tenant compte respectivement des variations de perméabilité et des variations d'épaisseur.

\section{GROUPE IV : Infiltrations au-dessous d'ouvra- ges d'art et à travers les digues hydrau- lique des puits.}

\section{1" Hydraulique des puits et galeries (7 rapports)}

L'hydraulique de l'écoulement permanent et non permanent à surface libre vers un puits est envisagée dans le premier des sept rapports se rapportant à ce sujet.

En ce qui concerne l'écoulement permanent, on peut écrire la relation de Dupuit :

$$
\mathrm{Q}=\mathrm{K} \pi \frac{\mathrm{H}^{2}-h^{2}}{\log (\mathrm{R} / r)}
$$

où $\mathrm{Q}$ et $\mathrm{K}$ sont le débit et la perméabilité, $\mathrm{H}$ et $h$ la hauleur de la surface libre aux distances $R$ et $r$ de l'axe du puits et $\mathrm{R}$ le rayon d'action $\mathrm{du}$ puits sous la forme :

$$
\left.\mathrm{H}-h=\frac{\mathrm{Q}}{2 \pi \mathrm{KH}} ; \frac{2 \mathrm{H}}{\mathrm{H}+h} \log \frac{\mathrm{R}}{r}\right)
$$

ou :

$$
\frac{2 \pi \mathrm{KH}}{\mathrm{Q}}(\mathrm{H}-h)=\frac{2 \mathrm{H}}{\mathrm{H}+h} \log \frac{\mathrm{R}}{r}=\mathrm{S}
$$

Un graphique expérimental (expériences et relaxation) donnant $\mathrm{S}$ en fonction de $(r / \mathrm{R})$ pour différentes valeurs de $\left(r_{0} / \mathrm{H}\right)$ et $\left(h_{0} / \mathrm{H}\right)\left(r_{0}\right.$ et $h_{0}$ étant respectivement le rayon et le tirant d'eau du puits) permet de tracer la surface libre.

Pour l'écoulement non permanent, après un rappel des différents travaux consacrés à cette question) : (Theis, Jacob, Boulton), une relation est proposée dans le cas d'une limite imperméable, verticale, circulaire, concentrique du puits.

"Quelques problèmes relatifs au rabattement de la nappe aquifère » sont examinés ensuite et en particulier :

- Le débit des puits n'atteignant pas la couche imperméable, pour lequel une relation approchée est proposée;

- Le débit et le rabattement des puits crépinés sur une hauteur inférieure à celle de la nappe.

Le problème des drains rayonnants dans une nappe en charge d'épaisseur constante a été examiné théoriquement pour une ou deux nappes de drains, dans l'hypothèse d'un rendement spécifique des drains, constant sur toute leur longueur.

Le problème d'une tranchée de longueur finie reposant sur un substratum imperméable horizontal a été ensuite étudié (hýpothèse de Dupuit généralisée par Forchheimer) pour un milieu 
infini et dans le cas de la proximité d'une rivière.

Diverses questions font ensuite l'objet de trois communications :

- Utilisation du calcul matriciel pour l'étude de l'action réciproque de plusieurs puits;

- Cas simples théoriques de recharge de nappes aquifères;

- Etude expérimentale de l'évolution dans le temps du débit d'un puits permettant la prévision du débit à un instant quelconque à l'aide d'un pompage de courte durée.

\section{$2^{\circ}$ Canalisation des Rivières (4 rapports).}

La canalisation des rivières de plaines est une préoccupation constante en Hongrie, pour le Danube et la Tisza. Les problèmes posés sont de deux sortes :

- Détermination des débits filtrant à travers les digues;

-- Implantation et dimensionnement du réseau de drainage éventuellement nécessaire dans les zones protégées par les digues.

Quatre études se rapportant à ces préoccupations traitent des sujets suivants :

-_- Etablissement d'une solution rigoureuse de l'écoulement vers un système de drains horizontaux, placés dans la couche superficielle reposant sur une couche de perméabilité nettement supérieure à la précédente et contenant de l'eau sous pression. L'alimentation des drains est due au mouvement ascendant provenant de la couche de base et à l'eau de pluie en provenance de la surface.

L'application des résultats théoriques est facilitée par de nombreux abaques.

-. Dimensions à donner aux tranchées de drainage situées à l'aval des digues de protection et sous-pressions sous la couche superficielle supposée étanche. Des relations très simples proposées ont été vérifiées par analogie électrique.

-.. Etude de l'écoulement dans une couche perméable d'épaisseur limitée recouverte de plusieurs couches de perméabilité beaucoup plus faible que la précédente.

- Etude de la répartition des sous-pressions sous les couches superficielles imperméables en régime permanent et non permanent. Sćcurité contre la rupture de ces couches.

\section{$3^{\circ}$ Filtration a travers les digues ( 3 rapports).}

Dans une très importanle étude est exposé l'ensemble des résultats obtenus à l'Institut $\mathrm{Hy}$ - draulique de Naples par l'exploitation de l'analogie de Hele Shaw. Ces résultats se rapportent aux divers points suivants :

Mouvements permanents :

- Influence de la capillarité;

- Milieux de perméabilités différentes.

Mouvement périodique de filtration d'une surface libre sans écoulement moyen.

Le second rapport expose les résultats d'observation sur un ouvrage existant tandis que le dernier généralise aux parements inclinés, les résultats obtenus pour les parements verticaux par Polubarinova-Kotchina et Barenblatt dans l'étude de la propagation, dans un milieu perméable d'une onde provoquée par une variation du plan d'eau amont. Ces résultats sont obtenus par intégration de l'équation de Boussinesq.

\section{4" Ridiaux de palplanches et terans Étan- CHES (3 rapports).}

Trois communications envisagent cette question de la manière suivante :

- Relation empirique donnant la perte de charge en fonction de la profondeur du rideau;

- Remous provoqué dans l'écoulement souterrain par un diaphragme;

- Influence des efforts hydrodynamiques sur la stabilité d'un rideau de palplanches formant mur de quai et correction correspondante à apporter à la méthode du cercle de glissement.

5" Diverses Questions SE RatTachant a LA FILTRATION ONT DONNÉ LIEU A LA PRÉSENTATION DE DEUX RAPPORTS.

Le premier expose les résultats de mesures ayant pour objet de déterminer la relation entre le rendement d'une couche aquifère (rapport entre le volume d'eau prélevé et le volume. total) et sa perméabilité.

La relation $\mathrm{K}=f$ ( $p$ active) est une parabole.

Le second rapport rend comple d'expériences ayant pour objet de définir l'efficacité d'étanchements à l'aide de bentonite.

La liste complète des 55 communications présentées au Congrès de Budapest, classées par "Questions », a paru dans La Honille Blanche, n" spécial B, décembre 1960 , pages 874 et 875 . 
D I S CUS S ION

president : M. Remenieras

M. Remexienas, obligé de se retirer pour une question de service, laisse la présidence à M. SERra.

M. Serra remercie M. Nougaro et M. Gerber pour cette communication.

M. Mahrutr demande à M. Nougaro des éclaircissements sur l'un des rapports présentés à la Conférence d'Hydraulique de Budapest : celui concernant l'étude des ondes de choc et son application aux canaux découverts. Les rapports ont-ils été publiés?

M. Novasno répond qu'il s'agit d'une étude théorique qui critique l'extrapolation des relations utilisées pour les conduites et donne une formule qui s'applique aux ondes de faible amplitude et ensuite aux ondes d'amplitude plus élevée.

Ces études ont été publiées par la Commission des Eaux de l'Académie des Sciences de Hongrie à Budapest. Pour se les procurer, il faut s'adresser à M. Nemeth, qui est le président de cette Commission et a été celui de ces Conférences.

Sur la demande de MM. HaEgelen et Remenieras, M. Novgaro dit que, seuls, les rapports généraux de ces conférences sont publiés en quatre langues, russe, hongrois, français et anglais.

M. Schneeibli demande à M. Nougaro de donner des explications sur le point suivant : dans le groupement $\mathbf{G}_{3}$, M. Novgaro a cité une communication parlant de la limite supérieure de validité de la loi de Darcy et a fait mention d'une limite inférieure.

M. Nougaro dit que cette deuxième partie aurait dû être traitée par M. Gerber et lit intégralement ce que celui-ci avait écrit, car lui-même n'en a fait qu'un résumé (prière de se reporter au mémoire de MM. Nougaro et Gerber : «Le second travail expose les résultats.., etc.).

M. Schneebeli s'est demandé quelle pouvait être, dans les écoulements liquides, la limite inférieure qui est très nette dans les écoulements gazeux : des Canadiens ont étudié des domaines intermédiaires où le régime d'écoulement visqueux passe au régime moléculaire. La limite de validité de la loi de Darcy est caractérisée par le rapport suivant:

Dimension des canaux capillaires ou dimension des grains

Libre parcours moyen des molécules gazeuses
L'auteur de la communication donne-t-il une explication physique à cette limite de validité pour les écoulements liquides, ou se borne-t-il à constater des résultats d'expériences?

M. Novgaro, pour donner une précision, lit un passage du texte : «En ce qui concerne la limite inférieure du domaine de validité de la loi de Darey, les auteurs soulignent avec insistance... $\geqslant$ (voir mémoire)

M. Schneebeli conclut que, dans les liquides comme dans les gaz, la limite inférieure de la loi de Darcy est due à des phénomènes moléculaires.

M. Remenieras pense que la loi de Darcy ne s'applique plus, lorsque les forces électriques deviennent prépondérantes ou tout au moins non négligeables vis-à-vis des forces de gravité. Cela est suggéré par diverses expériences d'électroosmose et d'électrophorèse.

M. SchneEbri ajoule que c'est le phénomène de polarisation électrique de la surface.

M. Houpeurt se demande si la limite inférieure de validité de la loi de Darcy ne serait pas liée à l'existence d'une structure moléculaire différente dans la masse du liquide et dans le voisinage immédiat de la surface solide, structure qui s'étend plus ou moins en profondeur et qui pourrait être plus ou moins altérée par les effets du gradient de pression. On sait que la viscosite des liquides au contact des solides est très supérieure à la viscosité moyenne dans la masse, ce qui est précisément expliqué par la structure moléculaire.

M. Bouvard indique que M. Nougaro a cité des études concernant la théorie de la turbulence. Ces études sontelles basées sur la théorie de Schmidt-Rouse ou partentclles de données différentes?

M. Novgaro répond que ces études sont différentes.

En réponse à une question qui lui est posée par un assistant, M. Nougaro précise que M. Gerber et lui-même n'ont pas traduit intégralement les communications, mais se sont contentés de les faire analyser par des persomnes connaissant la langue d'origine. 